\title{
Introducción al estudio del turismo a través del materialismo cultural ${ }^{1}$
}

\author{
Alejandro Palafox Muñoz \\ Lilia Zizumbo Villarreal ${ }^{* *}$ \\ Emilio Gerardo Arriaga Álvarez ${ }^{* * *}$ \\ Neptalí Monterroso Salvatierra****
}

\begin{abstract}
Resumen: Con el modelo de bienestar, los sectores económicos tuvieron un crecimiento sustantivo en México. Con el cambio del modelo económico, el sector servicios, sobre todo, el turismo, se ha consolidado como una actividad que homogeniza y funcionaliza el paisaje para facilitar la apropiación de los recursos naturales y culturales de las comunidades rurales para la expansión de la economía de libre mercado, con el objetivo de que permanezca el modo de producción y reproducción vinculado al capital, con lo que se contradice la sustentabilidad de la actividad. Para el análisis de dicha situación y sus implicaciones, se propone el uso de la estrategia materialista cultural (Harris, 1982) como base teórico-metodológica general fortalecida con las propuestas de Sauer (1925) para el conocimiento del paisaje natural y cultural, y de Cordero (2004) para el conocimiento de las formas de apropiación de la naturaleza y la cultura que utiliza la expansión capitalista. Con esta propuesta se espera coadyuvar en la identificación del proceso de apropiación, homogenización y funcionalización, vinculado al turismo.
\end{abstract} piación

Palabras clave: turismo, materialismo cultural, investigación crítica, apro-

\section{Introduction to the study of tourism through cultural materialism}

\footnotetext{
Abstract: With the well-being model, the economic sectors had a substantive growth in Mexico. With the change of the economic model, the services sector, mainly tourism, has consolidated as an activity that homogenizes and

* Universidad de Quintana Roo, Cozumel, México. Email: palafox@uqroo.mx

** Universidad Autónoma del Estado de México, Toluca, México. Email: lzv04@yahoo.com *** Universidad Autónoma del Estado de México, Toluca, México. Email: egearriaga@hotmail.com

**** Universidad Autónoma del Estado de México, Toluca, México. Email: n.monterrososalvatierra@gmail.com

${ }^{1}$ Agradecimientos: A la Universidad de Quintana Roo y al Programa de Mejoramiento del Profesorado (PROMEP) por el soporte económico para el desarrollo del proyecto de investigación: El turismo como eje de acumulación y factor de transformación del paisaje en Cozumel, México, así como a los árbitros por las recomendaciones realizadas para mejorar el artículo.
} 
functionalizes the landscape in order to facilitate the appropriation of the natural and cultural resources of rural communities for the expansion of the economy of free market, with the objective that the way of production and reproduction tied to its financing endures, with which contradicts the sustainability of the activity. For the analysis of this situation and its implications, the use of cultural materialistic strategy (Harris, 1982) is suggested, as general theoretical-methodological base, improved by the proposals of Sauer (1925) for the knowledge of the natural and cultural landscape, and of Cordero (2004) for the knowledge of the forms of appropriation of nature and culture that uses the capitalist expansion. With this proposal, helping in the identification of the appropriation process, homogeneization and functionalization, tied to tourism, is expected.

Key words: tourism, cultural materialism, critic research, appropriation

\section{Introdução ao estudo do turismo através do materialismo cultural}

Resumo: Com o modelo de bem-estar, os sectores económicos tiveram um crescimento sustantivo em México. Com a mudança do modelo económico, o sector serviços, sobretudo, o turismo, consolidou-se como uma actividade que homogeniza e funcionaliza a paisagem para facilitar a apropiação dos recursos naturais e culturais das comunidades rurais para a expansão da economia de livre mercado, com o objectivo de que permaneça o modo de produção e reprodução vinculado ao capital, com o que se contradiz a sustentabilidade da actividade. Para a análise de dita situação e seus envolvimentos, propõe-se o uso da estratégia materialista cultural (Harris, 1982) como base teórico - metodológica geral fortalecida com as propostas de Sauer (1925) para o conhecimento da paisagem natural e cultural, e de Cordeiro (2004) para o conhecimento das formas de apropiação da natureza e a cultura que utiliza a expansão capitalista. Com esta proposta espera-se coadyuvar na identificação do processo de apropiação, homogenização e funcionalização, vinculado ao turismo.

Palavras-chave: turismo, materialismo cultural, investigação crítica, apropiação

Recibido: 22.06.2009

Aceptado: 05.08.2009

$* * *$

\section{Prefacio para el estudio del turismo como eje de acumulación}

La expansión del capitalismo tiene su mayor énfasis con el modelo económico neoliberal, el cual tiende a absolutizar el mercado hasta transformarlo en el medio, método y fin del comportamiento humano (Compañía de Jesús; 1996). Asimismo, Touraine (2001) afirma que el neoliberalismo es un proceso de desarrollo capitalista y como tal es una forma de transformación del modelo, el cual se define como el proceso de autonomización e independización del mundo económico, del sistema económico con el resto de la sociedad, y el esfuerzo de este sistema para imponerse ante los demás mediante la construcción ideológica. 
Para ello, las organizaciones internacionales funcionan para tal fin, a través del Fondo Monetario Internacional (FMI), el Banco Mundial (BM) y la Organización para la Cooperación y el Desarrollo Económico (OCDE) mediante las políticas de reducción de los costos, disminución del gasto público y la flexibilización del empleo, es decir, la desregulación financiera. El programa neoliberal deriva su poder social del poder político y económico de los intereses de operadores financieros, industriales, políticos conservadores y socialdemócratas articulados por altos funcionarios financieros (Bourdieu, 1998).

Lo anterior se lleva a cabo por medio de los tratados de libre comercio, los cuales causan un desajuste en el progreso de los países capitalistas emergentes, así las sociedades enfrentan un estilo de desarrollo que se ha revelado ecológicamente depredador, socialmente perverso y políticamente injusto (Guimarães; 1994), mediante procesos y estructuras de dominación y apropiación de los recursos que se llevan a cabo a escala mundial imponiendo exigencias y estableciendo parámetros a favor de las naciones con mayor poder económico, militar y cultural (Ianni; 2001).

De acuerdo con Saxe-Fernández (2006), la economía de libre mercado ha contribuido al aumento de la pobreza y beneficiado a las empresas multinacionales a través de la eliminación de controles nacionales a la inversión extranjera; el fortalecimiento y formalización de los derechos de los inversionistas, de sus patentes y derechos de autor, a la par de un debilitamiento del consumidor; la eliminación de tarifas y requisitos de desempeño; y el establecimiento de tribunales internacionales que les permite a las trasnacionales realizar juicios contra gobiernos nacionales o locales en materia de leyes laborales, de salud o ambientales, de prestación de servicios que afecten negativamente o interfieran en el logro de las ganancias esperadas, es decir, la reproducción del capital.

Asimismo, el fenómeno de la internacionalización económica se caracteriza por el crecimiento en los flujos de mercancías, capital y tecnología, lo cual ocurre en contextos y relaciones imperialistas durante las últimas décadas y se ha enfatizado con la firma de los acuerdos para la explotación de recursos humanos y materiales de la periferia capitalista (Ídem). En este sentido, el papel del Estado ha girado en torno a reducir la inseguridad social en lugar de redistribuir la riqueza (Castel; 2001), con la finalidad de que los capitales se beneficien de las divisiones territoriales de la economía internacional, jugando con las jurisdicciones a fin de minimizar costos, evitar impuestos, regulaciones ambientales y obteniendo garantías políticas de estabilidad (Cox; 1994).

De esta manera, el sector económico propicio para la reproducción del modelo es el turismo, el cual se constituye en un nuevo orden a escala mundial, ya que éste fenómeno aparece vinculado a impulsos y motivaciones que podrían engendrar a escala social una movilidad generalizada (Lanfant, 1980), éste se encuentra financiado por la Organización Mundial del Turismo (OMT), el FMI, la Organización de las Naciones Unidas (ONU), 
entre otras, ya que las organizaciones internacionales atribuyen una gran importancia a los flujos turísticos internacionales por la cantidad de ingresos económicos que se generan, por tanto, dichas organizaciones intervienen directamente en la concepción y determinación de los flujos turísticos (Ídem), por ende, la promoción del turismo denota intereses puramente económicos, ya que los ingresos mundiales por esta actividad fueron del orden de 856 mil millones de dólares en 2007 (SECTUR, 2008), sin embargo entre Estados Unidos de Norteamérica, España y Francia (países desarrollados) reciben el 24.3\% de los ingresos (OMT, 2008), y la participación económica de los países latinoamericanos en el sector turístico sólo alcanza el 7.3\% del total mundial (WTTC, 2007).

El interés por el turismo comenzó después de la Segunda Guerra Mundial, al “consolidarse el poder de las naciones industrializadas sobre el resto del mundo, proceso simultáneo de mayores posibilidades socioeconómicas por parte de la población de las grandes metrópolis, el turismo quedó convertido en un recurso disponible en su oferta en el mercado” (Getino, 1991: 49), pero el propósito original es “articular el subdesarrollo al desarrollo de las sociedades más ricas por medio del turismo” (Lanfant, 1980: 17), encontrando respuesta al convertirse la actividad en un factor de desarrollo para las regiones económicamente débiles creando interdependencia entre las mismas, al constituirse una sociedad desarrollada en demandante y la subdesarrollada en oferente.

Aunado a esta dependencia, Sessa (1970) afirma que las sociedades en desarrollo están obligadas a realizar ciertas opciones en su economía para responder a las exigencias del mercado que han formado mediante una política adecuada del consumo privado y del tiempo libre, mantener y aumentar la demanda turística de los países industrializados, por tanto, en los países subdesarrollados que promovieron al turismo como estrategia para el desarrollo, se construyeron grandes centros turísticos que se beneficiaron de inversiones públicas en infraestructura e incentivos para la inversión privada, principalmente extranjera (Altés, 2006), mediante un modelo donde dominan las empresas transnacionales y se da prioridad a los resultados cuantitativos de la actividad -número de visitantes, gasto promedio, etc., con el propósito de diseminar la idea de que el turismo es un elemento catalizador del desarrollo a través de la administración privada y las fuerzas del mercado, otorgando preferencia a las empresas transnacionales de capital extranjero, a fin de garantizar a sus clientes que encontrarán las mismas comodidades y servicios que en Estados Unidos y Francia (Propín, et.al., 2004).

Por lo tanto, los recursos naturales y culturales juegan un papel para atraer la atención de inversionistas y la llegada masiva de extranjeros dando lugar a la creación de destinos en el área de América Latina y el Caribe como Acapulco, Cancún, Varadero, San Juan, Punta Cana, Isla Margarita, St. Kits, Ciudad de Panamá, Haití, entre otros; en donde se genera infraestructura para la actividad (hoteles, aeropuertos, restaurantes, etc.) y servicios complementarios para el desenvolvimiento de la actividad (hospitales, 
servicios bancarios, transporte, tour operadoras especializadas, etc.). En este sentido, destaca México como el país con mayor número de infraestructura hotelera en la región Latinoamericana al recibir 11.5 millones de turistas internacionales en 2007 (SECTUR, 2008), quedando claro el proceso de homogenización del espacio y del consumo fortalecido por la globalización económica (Yory, 2006).

El turismo actúa como el catalizador idóneo para la expansión del capital, a través de la inversión en infraestructura de servicio como hoteles, restaurantes, aeropuertos, carreteras, que aparecen en territorios con gran riqueza natural y cultural, pero que llegaron hasta que el destino de los recursos fue para atraer ingresos económicos, en lugar de proveer a la población de los insumos para su permanencia. Al respecto, el gobierno mexicano ha sinergizado el funcionamiento de la iniciativa privada por medio de la contrarreforma al artículo 27 constitucional abriendo "la puerta a la privatización y consiguiente desaparición del ejido” (Morales y García, 2002; 87). Así, el turismo se gesta en un vector de la globalización porque promueve los flujos financieros, de mercancías, de ideas y de personas a nivel global (Hiernaux, 1989), es decir, a la promoción del neoliberalismo y al proceso de globalización como instrumento político económico predominante, dejando en el mercado la organización y planificación de la actividad.

En México, este esquema de reproducción del capital a través del turismo comenzó en la década de los setenta y “constituye la traza para los próximos años privilegiando la inversión privada para la construcción y operación de empresas, y reduciendo la participación del Estado en el desarrollo de infraestructura y dotación de servicios públicos” (Pérez, et. al., 2009: 37), de esta manera el aparato burocrático justifica la instauración de la iniciativa privada como estrategia para la creación de empleos y la recepción de divisas por medio de la comercialización de los recursos naturales y culturales, mismos que son considerados bienes comerciales para la captación de nuevas empresas turísticas y la expansión de las ya existentes, "maximizando el beneficio económico para unos cuantos en detrimento de las colectividades asentadas en el medio rural” (Ídem).

Asimismo la incorporación de la fuerza de trabajo rural en el sector turístico ha tenido una serie de impactos en la sociedad, ejemplo de ello es la composición demográfica originado por los procesos migratorios (residenciales y laborales) que conlleva (Casado; 1999 y, Rodríguez y Warnes; 2002), también ha transformado la economía con la desaparición -casi en su totalidad- de las actividades productivas tradicionales (Vera; 1992). Por otra parte, la llegada de una nueva actividad económica generadora de divisas al corto plazo, ha contribuido al aumento de la tenencia de la tierra y el empleo, así como fuertes movimientos culturales al grado de la desaparición de la cultura local y de su sustitución por una cultura turística, cosmopolita y global (Torres; 2003: 65), así como la desaparición de la cultura ambiental que regulaba la forma y modo de relacionarse y de entender la naturaleza (Aledo; 2004) al ser modificado su paisaje. 
El desarrollo del turismo en México, así como la creación sociedades modernas ha traído consigo un fuerte impacto sobre los ecosistemas, ejemplo de ello es la pérdida de selvas a un ritmo de 93 mil hectáreas al año (SEMARNAT, 2005). Actualmente los principales factores que amenazan a las selvas y bosques son: el cambio en el uso del suelo, el crecimiento demográfico y de la infraestructura, los incendios forestales, la sobreexplotación de los recursos naturales y el cambio climático global (PNUMA, 2006). Para minimizar los efectos, se han creado las Áreas Naturales Protegidas, estrategia que ha surgido para contener el modelo depredador en boga, ya que el desarrollo del capitalismo ha traído consigo un fuerte impacto en la disminución de las selvas, ejemplo de lo anterior, en México se perdieron 1,322 mil hectáreas de selva principalmente entre 1993 y 2002, principalmente por la expansión de la frontera urbana, el crecimiento poblacional y la construcción de infraestructura (Becerra-Moreno, 1998 y SEMARNAT, 2005 citados por PNUMA, 2006).

Lo anterior ha repercutido en la transformación del paisaje (OSE; 2006 citado por Aledo; 2008) impactando directamente sobre el medio ambiente, principalmente en la desaparición del recurso vinculado a las costas, siendo sustituido por un nuevo entorno turístico por medio de la inversión para la modernización de las localidades rurales a través de la construcción de megadesarrollos, proyectos turísticos, hoteles, e instauración de equipamiento básico y complementario, con la finalidad de satisfacer la demanda del turismo extranjero, principalmente el norteamericano, en este sentido, en los últimos siete años se han invertido en México 19,373.99 millones de dólares en infraestructura portuaria, desarrollo inmobiliarios y turísticos, así como campos de golf, marinas, parques temáticos, hoteles, entre otros (SECTUR 2002 - 2008b). Todo ello genera nuevas identidades, las cuales buscan expresarse en los mitos y realidades que ofrece el espacio turístico (Chadefaud; 1987), a fin de integrarse a las nuevas experiencias que ofrece el turismo.

Las cifras precedentes son muestra del papel que desempeña el Estado para que "las empresas y conglomerados trasnacionales, en sus redes y alianzas, en sus planteamientos sofisticados, operando a escala regional, continental o global, disponen de condiciones para imponerse a los diferentes regímenes políticos, a las diversas estructuras estatales, a los diferentes proyectos nacionales" (Ianni; 2001: 94), es decir, a todos los procesos por medio de los cuales las sociedades de cada nación son incorporadas en una única sociedad mundial (Martin; 1990).

En los últimos cinco años, el estado de Quintana Roo ha recibido $11.84 \%$ de la inversión privada nacional y extranjera que ha ingresado al país (Ver Tabla 1), principalmente en el Proyecto Integralmente Planeado de Cancún, y los Megaproyectos Turísticos Riviera Maya y Costa Maya, mismos que son promovidos por el Fondo Nacional de Fomento al Turismo (FONATUR), el primero en la década de los setenta y los otros en los noventa. 


\section{Tabla 1. Inversión turística en Quintana Roo 2004 - 2008 (cifras en Millones de dólares).}

\begin{tabular}{|c|c|c|c|c|c|}
\hline Rubro / Aǹo & $\mathbf{2 0 0 4}$ & $\mathbf{2 0 0 5}$ & $\mathbf{2 0 0 6}$ & $\mathbf{2 0 0 7}$ & $\mathbf{2 0 0 8}$ \\
\hline Inversión turística & 427.62 & $\mathbf{8 1 8 . 1 3}$ & 393.5 & 1024.9 & 295.0 \\
\hline Total & & $2959.15=11.84 \%$ & \\
\hline
\end{tabular}

Fuente: Elaboración propia con información de Secretaría de Turismo (2002 - 2008b).

Lo anterior a través de "una política unisectorial y mecanismos que institucionalizan para tal fin, y de créditos que obtiene del financiamiento que le otorga la banca internacional en el marco de una economía subordinada” (Pérez y Carrascal, 2000: 145) a beneficiar la iniciativa privada, la cual ha impactado directamente en la modificación del territorio y en consecuencia en el detrimento de la sustentabilidad de las actividades económicas, sociales y ambientales de las comunidades donde el turismo se coloca como pilar para el ingreso de divisas y la generación de empleos, bajo la disyuntiva de cómo vivir mejor (mayor acceso a bienes y servicios) sin recurrir a los esquemas "con los que los países industrializados han explotado los recursos naturales y que hoy en día amenazan la continuidad de los ciclos productivos” (Alemán, 2005: 2 citado por Palafox y Segrado, 2008: 119).

Por ello, el presente artículo pretende ampliar los horizontes de la investigación turística por medio de la implementación del materialismo cultural en el estudio del turismo, de esta manera se emplea el soporte teórico y metodológico de Harris (1982), Sauer (1925) y Cordero (29004), para contribuir a la interpretación del proceso apropiación, homogenización y funcionalización del entorno vinculado a la actividad turística.

\section{Introducción al análisis del paisaje}

El término paisaje ha sido utilizado en diferentes áreas del conocimiento principalmente en la geografía, arquitectura y biología, así como en el arte y la literatura. Sin embargo, su concepción y empleo como categoría científica tiene su origen en la geografía, aunque las interpretaciones varían de acuerdo a la escuela y corriente que hace uso de la expresión (Mateo y Da Silva, 2007).

No obstante la diversidad de roles que desempeña el paisaje en el ámbito geográfico, destaca la necesidad de estudiarlo bajo la función que cumple éste como insumo para la reproducción del capital, teniendo como consecuencia su transformación para el desenvolvimiento de las actividades económicas, por lo que es necesaria su apropiación, homogenización y funcionalización.

De esta manera, se entiende por paisaje al territorio conformado por 
diferentes características, “el cual comprende un mosaico de espacios naturales y antrópicos que definen patrones espacio-temporales como resultado de las complejas interacciones entre factores físicos, biológicos, sociales y económicos” (Peña, et.al., 2006: 184). En este sentido, los cambios de uso del suelo para el impulso de las actividades primarias, secundarias y de servicios modifican la estructura, función y evolución del paisaje.

Con lo anterior, se puede afirmar que los cambios suscitados en el entorno son consecuencia del modelo económico en vigor, que se caracteriza por una urbanización e industrialización de los espacios, así como la intensificación de la agricultura y ganadería, además del incremento de infraestructura para el transporte, y la saturación de zonas turísticas (Rodríguez, 1997). De esta manera, el paisaje se constituye como uno de los fundamentos de las fuerzas económicas y sociales sobre los ambientes, permitiendo conocer las relaciones que tiene el hombre con el espacio a través de su transformación (Deffontaines, 1988).

Bajo este enfoque, Rodríguez (1997) propone tres perspectivas para el análisis del paisaje: como producto, recurso y representación. El primero entendido como resultado de las interacciones entre medio ambiente y desarrollo, es decir, entre procesos naturales e intervenciones humanas; en segundo término para el desarrollo económico: turismo, implantación de nuevos residentes y empresas, etc., y finalmente mediante la percepción que tiene el habitante de su propio medio, su identificación con el mismo y las formas de apropiación simbólica del espacio.

Por lo anterior, Lefebvre (1991) afirma que si se considera al paisaje como resultado de las intervenciones de los actores sociales y económicos, es decir mediante la expansión de las actividades económicas y la creación de asentamientos humanos, entre otros factores, denota relaciones sociales de producción, es decir, el espacio entra en las relaciones de producción y en las fuerzas productivas, por tanto, el territorio se dialectiza y se constituye en sustento de las relaciones económicas y sociales, ejemplo de este paisaje antrópico es la ciudad de Santiago de Chile, la cual creció a un ritmo del 10\% entre 1990 y 1995, y un 25\% la última década (Valencia e Ibarra, 2006), teniendo como resultado la sobreexplotación del paisaje natural y la utilización irracional de los recursos.

De esta manera, la apropiación, homogenización y funcionalización del espacio, se convierte en un proceso cultural, porque se crean bienes materiales, se construyen asentamientos, se fabrican elementos y materiales, métodos de gestión y control, de vigilancia y comunicación; se forman valores, modos de pensar, de percibir el mundo y creación de significados al crear espacios asilados, zonas residenciales y conjuntos mal conectados entre centro y periferia; finalmente la formación de espacios residenciales, comerciales, recreativos, para los marginados, entre otros (Cfr. Aguiló, 1999; Claval, 1999; Aponte, 2003; Cánoves, et.al., 2005).

Así, “la apropiación del paisaje se trata de una estrategia 
socioeconómica adaptada para los recursos de subsistencia” (Herrera, 1999: 222), lo cual ha modificado los usos del suelo, dando como resultado actividades económicas de tipo agrario, de extracción y prestación de servicios, lo cual ha mostrado el desmedido usufructo de los recursos naturales para el sostenimiento de los ingresos económicos, impactando severamente a través de la "homogenización espacial, pérdida de diversidad biológica y abandono rural” (Atauri, et.al., 1992: 417), entre otros.

En este mismo orden de ideas, el paisaje se transforma y se utiliza para la edificación de fábricas, plantaciones, zonas habitacionales y de esparcimiento para modelar globalmente el territorio (Artigues, 2006), es decir se funcionaliza con la finalidad de proporcionar los elementos necesarios para que las actividades económicas hagan uso del mismo y sea considerado productivo para la generación de riqueza, convirtiéndolo en mercancía capaz de ser utilizada, de esta manera "el poder económico que se irá concentrando en estos núcleos se constituirá en un elemento de presión tanto para la funcionalización del territorio interno como para la potencialización de la accesibilidad a través de los sistemas de transporte y comunicación” (Sánchez, 2007: 73).

\section{Paisaje y turismo}

América Latina cuenta con una gran diversidad biológica, sin embargo los cambios en el entorno y sus efectos son la consecuencia inevitable de un sistema socioeconómico y político que ha convertido a la naturaleza y a las personas en una mercancía (WRM, 2008). Bajo este panorama económico, los recursos naturales y culturales actúan como catalizador en el desplazamiento de las personas, por ello, la actividad turística contribuye con el 50\% del Producto Interno Bruto de la región al captar el 6\% del turismo internacional (PNUMA, 2006 y SECTUR, 2008).

Ejemplo de lo anterior es la pérdida del $45 \%$ de la diversidad biológica que se alberga en los bosques mexicanos, así como el 50\% de los manglares y el 33\% de los humedales, así como la mayor parte de las tierras agrícolas de las zonas semiáridas se encuentran deterioradas; las causas: cambio de uso del suelo, sobreexplotación, cambio climático y contaminación (CONABIO, 2008), características propias del turismo; es decir, el espacio por donde se mueven los hombres está modelado por sus actividades... es la consecuencia de la capacidad de los seres humanos para proyectar su futuro (Lefebvre, 1974; citado por Claval, 2002: 32).

Los cambios de uso del suelo acarrean consecuencias tales como la pérdida de usos tradicionales (cultivos, bosques, plantaciones, entre otros), lo que lleva consigo a la disminución de especies por falta de extensiones de refugio natural. Lo anterior es de gran importancia para la pérdida de biodiversidad, así mismo, la heterogeneidad espacial desempeña un papel importante en la conservación de las especies biológicas (Atauri, et.al., 2002). 
No obstante, la globalización ha fortalecido el interés por el medioambiente desconocido, con la finalidad de estimular al viajero a disfrutar del sentimiento de libertad e independencia, (Pavlovich, 2001), a través del reflejo de símbolos e imágenes de los diferentes escenarios (Urry, 1990), dando lugar al desarrollo del turismo y segundas residencias, "teniendo como resultado una pérdida económica, cultural y espacial” (Jennings, 2004: 27) de las localidades de los países emergentes.

En este sentido, la reproducción de esas motivaciones y necesidades contribuyen a la homogenización y funcionalización del espacio para la satisfacción de las mismas, produciendo nuevas relaciones de producción entre los agentes de las comunidades, originando "nuevas desigualdades locales y regionales para la definición de los flujos turísticos” (Camaratta, 2004: 4). Así mismo, se hacen evidentes las diferencias socioeconómicas y territoriales en la apropiación del espacio, "paisaje que obliga a repensar la relación entre las entidades territoriales nacionales, las estrategias y las organizaciones de las empresas en vías de la mundialización” (Santos, 2008:195), ya que con el afán de mostrar un escenario para el consumo internacional, los recursos naturales y culturales son modificados para su representación y comercialización, como en el caso del Acrópolis (Yalouri, 200, citado por Winter, 2004), del mismo modo ocurrió en Angkor, bajo la intervención de la UNESCO y la Organización Mundial del Turismo al promover el turismo cultural en la región asiática, teniendo como resultado un incremento anual del 30\% en el número de turistas extranjeros (Ministry of Tourism, 2000).

Los administradores y planificadores del turismo perciben el paisaje como un nuevo recurso para desarrollar la actividad económica, al igual que los promotores de las empresas turísticas (restaurantes, hoteles, agencias de viajes, tour operadoras, entre otros), para la creación de las distintas modalidades de turismo (aventura, cinegético, sol y playa, rural, etc.) dependiendo del entorno determinado, ya que los diversos tipos de turista buscan los escenarios que estén dispuestos a pagar para su satisfacción.

Del mismo modo, la localidad receptora percibe de modo diferente los elementos del territorio, ya que previo al turismo, el espacio tenía un uso agrario, el cual está lleno de significados otorgándole un valor diferente al entorno, mismo que fortalece su identidad "contrario a la homogenización de identidades en un mundo globalizado” (Tilley, 2006: 11) mediante la capitalización de lo histórico y folklórico de las clases populares, “ a las que arrancaron la memoria para luego vendérsela empaquetada con sentimiento nostálgico (Blanco, 2003).

De esta manera, nace la propuesta de estudiar el turismo como un elemento transformador del paisaje, el cual mediante su apropiación, homogenización y funcionalización para el desarrollo de la actividad económica, consolida al modelo económico neoliberal como un nuevo eje de acumulación para la reproducción del capitalismo. Para su análisis, se opta 
por la estrategia materialista cultural de Harris (1982), la cual servirá de base teórica y metodológica para el estudio del turismo, alimentada de las propuestas de Sauer (1925) y Cordero (2004) para una mejor comprensión del fenómeno.

\section{El materialismo cultural: estrategia de investigación}

El núcleo de los principios que guían el desarrollo de teorías en la estrategia de investigación materialista cultural, fue previamente enunciado por Marx (1970), al aseverar que “el modo de producción de la vida material determina el carácter general de los procesos de la vida social, política y espiritual” (Marx, 1970 [1859]: 21 citado por Harris, 1982: 71). En este sentido, Harris (1982) afirma que la desmitificación de la vida social se lleva a cabo mediante la destrucción de las ilusiones de origen social que falsean la conciencia humana.

Lo fundamental del materialismo cultural radica en que dicha estrategia “centra su atención en la interacción entre la conducta y el entorno físico, establecida a través del organismo humano y de su aparato cultural” (Harris, 2006: 571), de esta forma las prácticas del grupo y la ideología tienen conexión con las condiciones materiales, las cuales conforman sus prácticas.

Marx dio inicio con la estrategia de investigación al tratar de explicar la ley de la evolución cultural, la cual está conformada por: “1) la trisección de los sistemas socioculturales en base tecnoeconómica, organización social e ideología; 2) la explicación de la ideología y de la organización social como respuestas adaptativas a las condiciones tecnoeconómicas; 3) la formulación de un modelo funcionalista capaz de recoger los efectos entre todas las partes del sistema; 4) la previsión del análisis tanto de las variables que mantienen el sistema como de las que lo destruyen; 5) la preeminencia de la cultura sobre la raza” (Ídem: 209 - 210).

De esta manera, los materialistas culturales afirman que dicha estrategia tiene mejores resultados al estudiar las limitaciones materiales a las que está supeditada la existencia humana, es decir, a la necesidad de "producir alimentos, cobijo, herramientas y máquinas, y de reproducir las poblaciones dentro de unos límites establecidos por el medio ambiente” (Harris, 1998: 553). Esta condición materialista está separada de todo aspecto ideológico, mental o espiritual, ya que las carencias materiales impactan directamente en el modo en que las personas enfrentan la satisfacción de necesidades básicas en un hábitat específico.

De acuerdo con Dobbs (2003), el materialismo cultural enfatiza su análisis en las conductas observadas en el objeto de estudio, así como las ideas y los símbolos. Sin embargo, la particularidad de éste radica en que centra su atención en la comprensión de las causas que ocasionan el surgimiento de las diferencias y semejanzas de las sociedades y culturas. 
Acota al mencionar que dicha estrategia no se compromete en sí misma a explicar ningún tipo sociocultural específico o conjunto específico de instituciones. El punto de énfasis del materialismo cultural se centra en la causalidad en los sistemas culturales, más no en su aspecto ontológico. Así, el materialismo cultural es una estrategia de investigación, la cual "consiste en explicar el origen, mantenimiento y cambio del inventario global de diferencias y semejanzas socioculturales” (Harris, 1982: 42).

Para ello, Harris (1982 y 1998) sugiere el empleo de dos conjuntos diferentes de distinciones: en primera instancia, la distinción entre acontecimientos mentales y conductuales; posteriormente, la distinción entre acontecimientos de tipo emic y etic, a fin de aportar explicaciones causales a las diferencias y similitudes en el pensamiento y comportamiento que se encuentran entre los grupos humanos.

Así, los pensamientos y la conducta de los actores puede orientarse desde dos aspectos distintos: la de los propios participantes y la de los observadores; y para su presentación objetiva y subjetiva tanto del observador como del actor social, se opta por la utilización de los términos emic y etic, a fin de no emplear los términos objetivo y subjetivo que puedan ocasionar confusión, y con las operaciones de tipo emic el observador trata de esclarecer las categorías y reglas para pensar y actuar como poblador; y las operaciones de tipo etic son para generar categorías desde un punto de vista científico sobre las causas y semejanzas socioculturales (Ídem, 1982).

El sistema sociocultural está constituido por la infraestructura, la estructura y la superestructura, la primera entendida como las características tecnológicas, económicas, ambientales y demográficas para la subsistencia-modo de producción y reproducción; la segunda, es la forma en que la que población se compone para satisfacer sus necesidades, refiriéndose a la economía nacional y economía política (modo de producción social), en el cual la comunidad se organiza y reproduce para dar continuidad al sostenimiento de lo que la sociedad es (y conformar el modo de reproducción económico); finalmente el último componente concentra los elementos simbólicos e ideológicos (modo de producción político e ideológico), el cual da pie al modo de reproducción social para conservar la vida social, la convivencia familiar, educación, entre otros a fin de integrar el modo de producción cultural, el cual está formado de ideas y acciones.

Con base en lo anterior, Harris y Ross (1999) resume al mencionar que la infraestructura consiste en el modo de producción y modo de reproducción, es decir que las sociedades deben satisfacer sus necesidades básicas para la manutención mediante el uso de la tecnología y la especificidad del hábitat; por otra parte, su reproducción mediante la limitación o mantenimiento del tamaño de la población, por consiguiente la existencia de un modo de producción conductual etic y un modo de reproducción conductual etic (Harris, 1982); además de mantener las relaciones con los integrantes de su grupo y con otras sociedades. Sin embargo, es pertinente considerar la problemática de equilibrar los procesos de tipo económico, principal- 
mente aquellos que “distribuyen el trabajo y sus productos materiales entre individuos y grupos” (Ídem: 68), lo que redunda entre otras cosas en el acaparamiento del capital.

De esta manera, la estructura se constituye por la economía doméstica y la economía política; y la superestructura, entendida como las creencias y las prácticas estéticas, simbólicas, filosóficas y religiosas. La propuesta estructural de Harris (1982), se fundamenta en las constantes biológicas y psicológicas de la naturaleza humana y la distinción entre pensamiento y conducta, así como entre las visiones emic y etic, apoyadas de los datos demográficos, económicos, tecnológicos y ambientales.

Lo anterior da pie a la formación de grupos locales o globales, entendidas como economías domésticas y economías políticas conductuales etic (Ídem), es decir, la organización en el intercambio y consumo básico en la localidad y entre los municipios y regiones. Finalmente, el comportamiento de la sociedad se puede simplificar en sus productos y servicios recreativos -entre ellos el turismo-, deportivos y estéticos de tipo etic. Por tanto, la superestructura conductual constituye una etiqueta adecuada para este sector etic de implantación universal (Ídem). La superestructura mental emic es entendida como las categorías, reglas, principios, filosofía, creencias y valores sobre el comportamiento de los individuos.

Bajo el contexto anterior, Harris (1982) ratifica que el materialismo cultural afirma la prioridad estratégica de los procesos y condiciones de tipo etic y conductuales sobre los de índole emic y mental, y de los procesos y condiciones infraestructurales sobre los estructurales y superestructurales; sin embargo, no descarta la posibilidad de que los componentes emic, mentales, superestructurales y estructurales alcancen cierto grado de autonomía con respecto a la infraestructura conductual etic.

El aparato tecnológico ha contribuido a que dicho proceso de producción y reproducción tenga la capacidad de elevar o disminuir su valor, pero, la tecnología también está sujeta a las leyes naturales, las cuales son inalterables y se constituyen como el umbral de su constante cambio y transformación, en consecuencia se convierte en el indicador y monitor de dicha variabilidad. De esta manera, la infraestructura se establece como el espacio de interacción entre cultura y naturaleza, es precisamente ahí en “donde las restricciones ecológicas, químicas y físicas a que está sujeta la acción humana con las principales prácticas socioculturales destinadas a intentar superar o modificar dichas restricciones” (Ídem: 73). Por lo anterior, el determinismo infraestructural establece la búsqueda de las semejanzas y diferencias de los sistemas socioculturales.

En este orden de ideas, se confirma que las fuerzas materiales de producción entran en conflicto con las relaciones de producción existentes y comienza "el periodo de revolución social durante el cual toda la inmensa superestructura se transforma con mayor o menor rapidez” (Harris, 2006: 200). Así mismo, los factores demográficos contribuyen a explicar la ex- 
pansión histórica de las fuerzas productivas del modo de producción cuyo impacto sobre las estructuras sociales y la ideología es tan significativo como el modo de reproducción (Ídem, 1982).

De esta manera, el materialismo cultural consta de tres componentes del sistema y cualquier cambio en alguno de ellos conduce a modificaciones en los restantes, entonces la consecuencia más probable de alguna innovación es una retroalimentación negativa del sistema, dado que esta estrategia de investigación sostiene que los cambios apoyados en los modos de producción y reproducción etic y conductuales suelen impactar en la economía doméstica, política, prácticas y creencias, a diferencia de un efecto inverso (Cfr. Harris, 1982: 89).

La estrategia de investigación materialista cultural será un elemento importante para el estudio del turismo, sin embargo es necesario agregar elementos que fortalezcan distintivamente al objeto de estudio, debido a que como se ha referido con anterioridad, el materialismo cultural busca las semejanzas y diferencias de los sistemas culturales, sin embargo para ahondar en las causas de la modificación del paisaje y las formas de acumulación capitalista en el objeto de estudio hace imperante el uso de otros elementos que se presentan a continuación.

\section{El turismo desde la perspectiva materialista cultural}

No obstante la complejidad que implica la investigación turística, y dado que no es únicamente un fenómeno sociocultural que involucra las relaciones entre los individuos sino también las implicaciones que resultan de la interacción con el entorno económico y ambiental, por lo cual es pertinente distinguir las características de la sociedad para obtener un panorama general del contexto (Wagley y Harris, 1974).

Con el modelo de bienestar, los sectores económicos tuvieron un crecimiento sustantivo en México, y con el cambio del modelo económico, el sector servicios, sobre todo, el turismo, se ha consolidado como una actividad que se apropia, homogeniza y funcionaliza el paisaje para facilitar la apropiación de los recursos naturales y culturales de las comunidades rurales para la expansión de la economía de libre mercado, con el objetivo de que permanezca el modo de producción y reproducción vinculado al capital, oponiéndose a la sustentabilidad de la actividad.

Para el análisis de dicha situación y sus implicaciones, se utiliza la estrategia materialista cultural (Harris, 1982) como base teórica general, y los elementos metodológicos proporcionados por las propuestas de Sauer (1925) para el conocimiento del paisaje natural y cultural, y de Cordero (2004) para el conocimiento de las formas de apropiación de la naturaleza y la cultura que utiliza la expansión capitalista, con el propósito de identificar el proceso de apropiación, homogenización y funcionalización, vinculado el turismo (Ver Figura 1). 
Figura 1. El turismo a través del materialismo cultural.

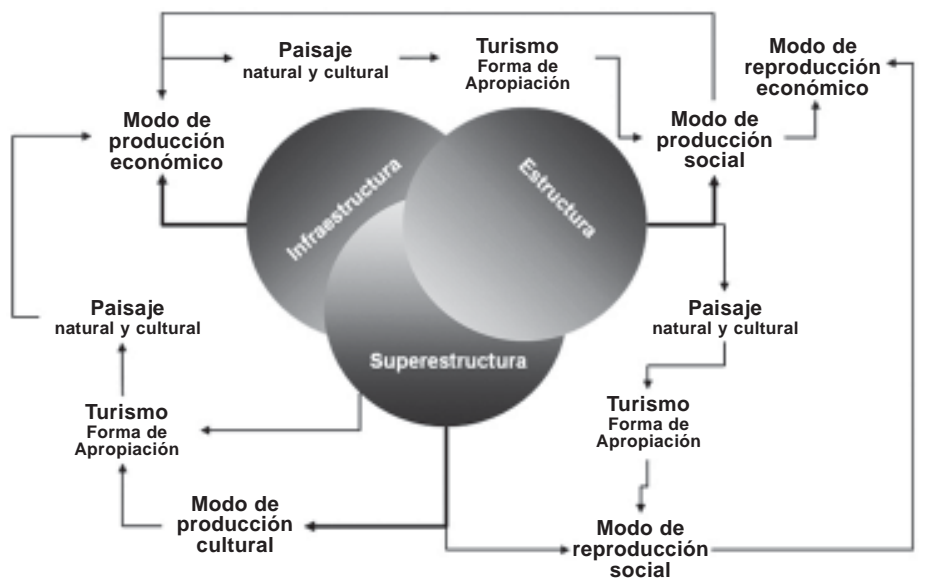

Fuente: Elaboración propia.

El turismo necesita del espacio natural y cultural para desarrollarse, y de acuerdo con Sauer (1925), el paisaje es un área conformada por una asociación de distintas formas, tanto físicas como culturales, es decir, "el contenido del paisaje se encuentra por tanto en las cualidades físicas del área que son significantes para el hombre y en las formas de uso del área, en hechos de sustento físico y hechos de la cultura humana” (Ídem: 23), ya que la naturaleza dotó al mundo de tierras fértiles y variedad de recursos que carecían de valor por sí mismos (Harris, 1973), así, la naturaleza y la cultura son elementos determinantes del desarrollo del turismo en forma aislada o combinada. Así mismo, el paisaje no es una escena individual sino el agregado de características generales (Sauer, 1925).

De esta manera, la descripción del paisaje natural y cultural permitirá conocer como se ha transformado el entorno a través del tiempo y ver sus cambios al pasar de una actividad primaria a otra terciaria, así como el proceso de homogenización, funcionalización y apropiación del entorno, por ello se seleccionan las cualidades del paisaje que son útiles para la investigación y se descartan aquellos rasgos que no son significantes para el hombre y su actividad económica, es decir, las cualidades del físicas y culturales del paisaje, son aquellas que tienen valor de hábitat actual o potencial y su valor de uso en hechos de sustento físico -la suma de recursos naturales- y de cultura humana -vivienda, lenguaje y costumbres de un grupo (Ídem).

El desarrollo turístico ha tenido un amplio crecimiento en los países Latinoamericanos y se ha convertido en un eje económico -de acumula- 
ción- por su capacidad para la generación de divisas y empleo; sin embargo el turismo desnuda el grado de desarrollo de una comunidad y expone el subdesarrollo de otras, a través de la crudeza de las desigualdades internas que caracterizan a un país o región, recordando así la imagen imperialista esclavista, por la diferencia social en términos de acumulación de la riqueza y por ende de poder (Cordero, 2004).

La actividad turística se torna en componente reproductor del modo de producción, ya que "el capital que se abre paso estructurando bajo su funcionamiento las nuevas formas de actividad económica” (Ídem: 104).

Esta lógica capitalista, se manifiesta mediante la incesante compra y venta de mercancías por medio de los mecanismos de libre mercado, dichas mercancías provienen de la explotación directa de la naturaleza. Al respecto, el PNUMA (2000) manifiesta que el sistema capitalista y su modelo consumista ignoran la variable ambiental, también sostiene que el turismo está estrechamente vinculado con la presencia de severos impactos ambientales como lo son: la sobreexplotación de las fuentes acuíferas, la contaminación de ríos y mares, la sobreconstrucción de infraestructura turística y la destrucción de los recursos naturales. Así, el auge del capitalismo y la expansión del turismo, se contribuye a la transformación del paisaje a fin de continuar con la productividad y los servicios que ha brindado éste en su nuevo rol económico (SER, 2004), saturado de la ideología neoliberal, misma que plasma su política medioambiental aplicando parámetros de sustentabilidad congruentes con la reproducción del capital.

Dicha contradicción entre economía y naturaleza, se hace evidente al convertirse en una contradicción política y social. Por lo que se analizará el turismo desde la perspectiva de la economía política, la caracterización de la naturaleza de acuerdo al interés turístico y las formas de apropiación del espacio, así como la conservación ambiental.

Así, el turismo desde la óptica materialista cultural está constituido en primer término por la infraestructura, misma que se encuentra integrada por el modo de producción (capitalista), el cual basado en la lógica de la acumulación y reproducción del capital mediante la alienación del funcionamiento del Estado, ya que "la expansión de los viajes es una fuerza de primer orden en el proceso de globalización” (Rodríguez, 2008: 1), con el propósito de apropiarse, homogenizar y funcionalizar el paisaje para el desarrollo de la actividad, lo cual ha "creado una creciente interdependencia, sino también a marcadas desigualdades internacionales” (Guimarães, 2003: 10), por tanto, la economía mundial se encuentra desnivelada y sus características propias son la "concentración del capital y la generación de tecnología en los países desarrollados, y su fuerte gravitación en el comercio de bienes y servicios” (Ídem).

Por ello, el papel desmesurado de las organizaciones trasnacionales en la difusión mundial de patrones culturales o del movimiento global de mercancías a la par del flujo informativo (Aguirre, 2005: 37), a fin de sos- 
tener el modo de producción, mediante procesos de apropiación, homogenización y funcionalización del paisaje.

La estructura está determinada por la infraestructura, misma que fija las directrices de la economía política y de la economía local, teniendo como estrategia para la expansión del modo de producción el desarrollo del turismo internacional como pauta dominante del crecimiento económico y su encadenamiento, a pesar de las implicaciones negativas sociales, económicas y ambientales del mismo (Stonich, 1998), que tratan de ser solventadas -discursivamente en el caso de México- a través de la sustentabilidad como pilar de la economía política; por ello se adhiere al análisis el enfoque de la ecología política para confrontar los desafíos de la globalización, ya que esta visión debe partir de la base de que un problema ecológico muestra disfunciones de carácter político y social (Cfr. Guimarães, 2003b, Alimonda, 2002, Lipietz, 2002 y O’Connor, 2002), ya que las instituciones son incapaces de enfrentar el reto de la escasez ecológica y ambiental, por tanto para superar la crisis es necesario tomar decisiones políticas; "y en ese proceso algunos intereses serán favorecidos más que otros” (Guimarães, 2003b: 12).

En este sentido, la ecología política se considera como un acercamiento interdisciplinario para el estudio de las interacciones del ser humano con el ambiente, particularmente aquellas asociadas con el desarrollo económico del tercer mundo (Bryant, 1992, Peet y Watts, 1993). En general, "el análisis de la ecología política consiste en una explicación integral de las interacciones hombre con el entorno natural vinculado a través de diferentes escalas desde lo global y local” (Blaikie and Brookfield, 1987 citado por Stonich, 1998: 29), puntualiza en las relaciones de poder de los actores sociales con respecto al acceso y manejo de los recursos naturales, así como sus niveles de poder con y entre ellos (Peet y Watts, 1993).

Finalmente, el tercer aspecto es la superestructura influenciada por los elementos infraestructurales y estructurales, confecciona gran parte del quehacer social y forma parte importante en la conformación del capital simbólico; por lo que es importante interpretar el pensamiento y acción de la población receptora con respecto a su racionalidad ambiental, la cual está constituida por un "conjunto de criterios para la toma de decisiones de los agentes sociales, para orientar las políticas, normar los procesos de producción y consumo, y legitimar las acciones y comportamientos de diferentes actores y grupos sociales para alcanzar el desarrollo sustentable" (Leff, 2004: 212), dado que el turismo está profundamente relacionado con los recursos naturales y culturales de las localidades, y la explotación de los mismos está estrechamente vinculada con la racionalidad capitalista, "razón como desarrollo desenfrenado de la productividad, conquista de la naturaleza y ampliación de la masa de bienes” (Marcuse, 1972: 207 citado por Leff, 2004: 213), lo que ha llevado a las comunidades a transformar su visión con respecto al aprovechamiento de los recursos naturales y culturales, modificando el paisaje y transformándolo para la permanencia de las actividades económicas que sustentan y expanden el modo de producción, 
legitimando su dominio mediante la apelación "a la creciente productividad y creciente dominación de la naturaleza que también proporciona a los individuos una vida más confortable” (Habermas, 1986: 56).

\section{Reflexiones finales}

El turismo se constituye como un elemento propagador del modo de producción de capitalista, el cual se apropia de los recursos naturales y culturales constituir su oferta, así mismo, homogeniza y funcionaliza el paisaje para el desarrollo y permanencia de la actividad económica al ofrecer bienes y servicios estandarizados para el disfrute de las sociedades capitalistas y por ende la reproducción del modelo.

El proceso de apropiación, homogenización y funcionalización forman un modelo cultural que coadyuva a la permanencia del modelo económico en vigor, por ello, el materialismo cultural provee las categorías teórico - metodológicas para el estudio del turismo como nuevo eje de acumulación.

Sin embargo, la estrategia materialista cultural de Harris (1982) se fortalece con los elementos metodológicos de Sauer (1925) y Cordero (2004) para brindar un claro entendimiento de la dinámica totalizadora que el modo de producción capitalista emplea para la transformación del paisaje, así como para su apropiación, homogenización y funcionalización a través del turismo.

El rol del turismo como eje de acumulación se presenta con mayor énfasis en los países capitalistas emergentes, para el caso latinoamericano, México se constituye como el espacio propicio para su estudio, por su localización estratégica y vínculos económico - comerciales con Estados Unidos de Norteamérica, lo que ha ocasionado la inserción de empresas transnacionales en el territorio mexicano, así como la desaparición de la biodiversidad principalmente en el espacio costero.

Es importante enunciar que la mayoría de las inversiones realizadas en México en el rubro turístico son de países capitalistas desarrollados, mismos que son concentran 1/4 de los ingresos por turismo internacional, con lo que se afirma que la actividad turística se constituye como eje de acumulación generando a su vez dependencia económica hacía los países emergentes. 


\section{Bibliografía}

Aguiló Alonso, M. (1999), El paisaje construido. Una aproximación a la idea de lugar, Colegio de Ingenieros, Caminos y Puertos, Madrid.

Aguirre Rojas, C. A. (2005), "Prefacio. Immanuel Wallerstein y la perspectiva crítica del análisis de los sistemas - mundo”, en: La crisis estructural del capitalismo, Editorial Contrahistorias, Distrito Federal.

Aledo, A. (2008), “De la tierra al suelo: la transformación del paisaje y el nuevo turismo residencial”, en: Arbor, No. 729, pp. 99 - 113, Consejo Superior de Investigaciones Científicas, Madrid.

Idem (2004), “Turismo y desarrollo: una reflexión sobre las teorías posdesarrollo”, en: Antonio Miguel Nogués (ed.), Cultura y desarrollo, Signatura Demos, Sevilla.

Alemán Santillán, T. (2005), “Desarrollo sustentable: teoría y práctica”, en: Ecofronteras, No. 24, pp. 2 - 8, Chetumal.

Alimonda, H. (2002), “Introducción: política, utopía, naturaleza”, en: Héctor Alimonda (comp.), Ecología política, naturaleza, sociedad y utopía, CLACSO, Buenos Aires.

Altés, C. (2006), El turismo en América Latina y el Caribe y la experiencia del BID, Banco Interamericano de Desarrollo, Washington.

Aponte García, G. (2003), “Paisaje e identidad cultural”, en: Tabula Rasa, No.1, pp. 153 - 164, Universidad Colegio Mayor de Cundinamarca, Bogotá.

Artigues Bonet, A. A. (2006), "Funcionalización turística y proceso de urbanización en la isla de Mallorca”, en: A. Artigués Bonet (ed.), Introducción a la geografía urbana de las Illes Balears, Universitat de les Illes Balears, Palma de Mallorca.

Atauri, J. A., C. L. De Pablo, P. Martín De Agar, M. F. Schmitz, F. M. Ugarte y F. D. Pineda (1992), “Tendencias de cambio reciente en el paisaje: relación con los factores socioeconómicos en la Reserva de la Biosfera de Urdaibai (Bizkaia)”, en: Cuadernos de Sección - Historia, No. 20, pp413 - 434, Eusko Ikaskuntza, Donostia.

Becerra-Moreno, A. (1998), “Conservación de suelos y desarrollo sustentable, ¿utopía o posibilidad en México?”, en: Terra, Vol. 16, No. 2, pp: 173 - 179,_Universidad Autónoma de Chapingo - Sociedad Mexicana de la Ciencia del Suelo A.C., Chapingo.

Bellamy Foster, J. (2000), La ecología de Marx: materialismo y naturaleza, Ediciones El Viejo Topo, Mataró. 
Blaikie, P. y H. Brookfield (1987), Land Degradation and Society, Methuen, Londres.

Blanco Marín, C. J. (2008), “Marxismo ecosocialista”, en: Nómadas, Vol. 1, No. 17, pp. 171 - 180, Universidad Complutense de Madrid, Madrid.

Bouridieu, P. (1998), “The essence of Neoliberalism: Utopia of endless explotation”, en: Le Monde Diplomatique, Diciembre 8, Paris.

Bryant, R.L. (1992), "Political Ecology: An Emerging Research Agenda In Third World Studies”, en: Political Geography, Vol. 11, No. 1, pp. 12 - 36, Elsevier, Londres.

Camaratta, E. B. (2004), Misiones: Turismo, paisaje en la frontera con Paraguay y Brasil, Universidade Federal do Rio de Janeiro, Río de Janeiro.

Cánoves Valiente, G., L. Herrera Jiménez y M. Villarino Pérez (2005), Turismo rural en "España: paisajes y usuarios, nuevos usos y nuevas visiones”, en: Cuadernos de Turismo, No. 15, pp. 63 - 76, Universidad de Murcia, Murcia.

Casado Díaz, M. (1999), “Socio-demographic impacts of residential tourism: a case study of Torrevieja, Spain”, en: The International Journal of Tourism Research, Vol. 1, No. 4, pp. 223 -237, John Wiley and Sons Ltd., Hoboken.

Castel, R. (2001), “Empleo, exclusión y las nuevas cuestiones sociales”, en: Desigualdad y globalización: cinco conferencias, Universidad de Buenos Aires, Buenos Aires.

Chadefaud, M. (1987), Aux origines du tourisme dans les pays de l'Adour, du mythe a_l'space: un essai de ge_ographie historique, l'Universite de Pau, Cedex.

Claval, P. (1999), A geografía cultural, Editorial da Universidade Federal de Santa Catarina, Santa Cartarina.

Idem (2002), "El enfoque cultural y las concepciones geográficas del espacio”, en: Boletín de la A.G.E., No. 34, pp. 21 - 39, Asociación de Geógrafos Españoles, Madrid.

Compañía De Jesús (1996), El neoliberalismo en América Latina, Universidad Iberoamericana, Distrito Federal.

CONABIO (2008), La biodiversidad biológica de México, Consejo Nacional para el Conocimiento y Uso de la Biodiversidad, Distrito Federal.

Cordero, A. (2004), Nuevos ejes de acumulación y naturaleza: el caso del turismo, FLACSO, Curridabat. 
Cox, R. W. (1994), “Global Restructuring: Making Sense of the Changing International Political Economy”, en: Richard Stubbs y Geoffrey Underhill (eds.), Political Economy and Changing Global Order, Cambridge University Press, Cambridge.

Deffontaines, J. P. (1988), “L’analyse de la petite región: de marches et notions”, en: Marcel Jolivett (ed.), Pour une agricultura diversifié, L’Harmattan, Paris.

Dobbs, M. R. (2003), Beyond Cultural Materialism: Assessing the Theoretical Contributions of Marvin Harris, Tesis de Mestría en Antropología, California State University, Fullerton.

Getino, O. (1991), El desarrollo del turismo en América Latina, LIMUSA, Distrito Federal.

Guimarães, R. P. (1994), “El desarrollo sustentable ¿propuesta alternativa o retórica neoliberal?”, en: Revista Eure, Vol. XX, No. 61, pp. 41 - 56, Universidad Complutense de Madrid, Madrid.

Idem (2003), “Tierra de sombras: desafíos de la sustentabilidad y el desarrollo territorial y local ante la globalización corporativa”, en: Serie Medio Ambiente y Desarrollo, No. 67, Comisión Económica para América Latina y el Caribe (CEPAL), Santiago de Chile.

Harris, M. (1998), Antropología cultural, Alianza Editorial, Madrid.

Idem (2006), El desarrollo de la teoría antropológica: una historia de las teorías de la cultura, Siglo XXI, Distrito Federal.

Idem (1982), El materialismo cultural, Alianza Universidad, Madrid.

Idem (1973), Raza y trabajo en América, Ediciones Siglo Veinte, Buenos Aires.

Harris, M., y E. B. Ross. (1999), Muerte, sexo y fecundidad, Alianza Editorial, Madrid.

Habermas, J. (1986), Ciencia y tecnología como ideología, Tecnos, Madrid.

Herrera Wassilowsky, A. (2003), "Patrones de asentamientos y cambios en las estrategias de ocupación en la cuenca sur del río Yanamayo”, en: Arqueología de la sierra de Ancash: propuestas y perspectivas, Instituto Cultural Runa, Lima.

Hiernaux, D. (1989), Teoría y praxis del espacio turístico, Universidad Autónoma Metropolitana - Xochimilco, Distrito Federal. 
Ianni, O. (2001), “Las ciencias sociales y la modernidad - mundo”, en: Desigualdad y globalización: cinco conferencias, Universidad de Buenos Aires, Buenos Aires.

Jennings, S. (2004), “Landscape Sensitivity and Tourism Development”, en: Journal of Sustainable Tourism, Vol. 12, No. 4, pp. 271 - 288, Taylor \& Francis Group, Londres.

Lanfant, M. F. (1980), “Introducción. El turismo en el proceso de internacionalización”, en: Revista Internacional de Ciencias Sociales, Vol. XXXII, No. 1, pp. 14 - 45, UNESCO, Paris.

Lefebvre, H. (1974), La production de l' espace, Anthropos, Paris.

Idem. (1991), The production of the space, Wiley - Blackwell, Londres.

Lefeuvre, J. C. (1989), “L’écologie ne peut plus être une refléxion sur la nature”, en: Marcel Juvillet (ed.), Du rural à l’environnement, L’Harmattan, Paris.

Leff, E. (2004), Racionalidad ambiental, Siglo XXI, Distrito Federal.

Lipietz, A. (2002). “A Ecologia Política, solução para a crise da instância política?”, Héctor Alimonda (comp.), Ecología política, naturaleza, sociedad y utopía, CLACSO, Buenos Aires.

Marcuse, H. (1972), Industrialization and capitalism in the work of Max Weber, Penguin, New York.

Martin, A. (1990), “Globalization, Knowledge and Society”, en: Martin Albrow y Elizabeth King (eds.), Globalization, Knowledge and Society: Readings from International Sociology, Sage Publications, Londres.

Marx, K. (1970), A contribution of the Critique of Political-Economy, International Publishers, New York.

Idem (1990), Introducción general a la crítica de la economía política / 1857, Siglo XXI, Distrito Federal.

Marx, K. y F. Engels (2008), Ideología alemana, Colofón, Distrito Federal.

Mateo Rodríguez, J. M. Y E. V. Da Silva (2007), “La geoecología del paisaje como fundamento para el análisis ambiental”, en: REDE, Vol. 1, No. 1, pp. 77 - 98, Universidade Federal do Ceará, Benfica - Fortaleza.

Ministry Of Tourism (2000), Cambodia Tourism Statistical Report 2000, Ministry of Tourism, Camboya. 
Morales, J. y A. García De Fuentes (2002), “Dimensión regional de la recesión”, en: México en el primer año del gobierno de Vicente Fox, Miguel Ángel Porrúa - Universidad Autónoma de Zacatecas, Distrito Federal.

O’Connor, J. (2002), “¿Es posible el capitalismo sostenible?”, en: Héctor Alimonda (comp.), Ecología política, naturaleza, sociedad y utopía, CLACSO, Buenos Aires.

OMT (2008), “Barómetro Turístico”, en: Barómetro OMT, Vol 6, No. 2, Organización Mundial del Turismo, Madrid.

OSE (2006), Sostenibilidad en España 2006, Mundi-Prensa Libros, Madrid.

Palafox Muñoz, A. y R. Segrado Pavón (2008), “Capacidad de carga turística: alternativa para el desarrollo sustentable de Cozumel”, en: Turismo \& Desenvolvimento, No. 10, pp. 109 - 120, Universidad de Aveiro, Aveiro.

Pavlovich, K. (2001), “The Twin Landscapes of Waitomo: Tourism Network and Sustainability through the Landcare Group”, en: Journal of Sustainable Tourism, Vol. 9, No. 6, pp. 491 - 504, Taylor \& Francis Group, Londres.

Peet, R. y M. Watts (1993), “Introduction: Development Theory ad Environment in an Age of Market Triumphalism”, en: Economic Geography, Vol. 69, No. 3, pp. 227 - 253, Clark University, Worcester.

Peña Cortés, F., G. Robledo, K. Hermosilla, E. Haustein, C. Beltrán, R. Schlatter y J. Plata (2006), “Dinámica del paisaje para el periodo 1980 2004 en la cuenca costera del Lago Budi, Chile. Consideraciones para la conservación de humedales”, en: Ecología Austral, No. 16, Diciembre, pp. 183 - 196, Asociación Argentina de Ecología, Buenos Aires.

Pérez Ramírez, C., L. Zizumbo Villarreal y N. Monterroso Salvatierra (2009), “Turismo e identidad de resistencia; la oposición local a proyectos turísticos en el Parque Nacional Nevado de Toluca, México”, en: Estudios y Perspectivas en Turismo, Vol. 18, No. 2, pp. 36 - 52, Centro de Investigaciones y Estudios Turísticos, Buenos Aires.

Pérez Villegas, G. Y E. Carrascal (2000), “El desarrollo turístico en Cancún, Quintana Roo y sus consecuencias sobre la cubierta vegetal”, en: Investigaciones Geográficas, No. 43, pp. 145 - 156, Universidad Nacional Autónoma de México, Distrito Federal.

PNUMA (2000), GEO - América Latina y el Caribe: Perspectivas del medio ambiente, Observatorio del Desarrollo - Universidad de Costa Rica, San Jose.

Idem (2006), Iniciativa Latinoamericana y Caribeña para el Desarrollo Sostenible, Indicadores de seguimiento: México 2005, Programa de las 
Naciones Unidas para el Medio Ambiente - Secretaria de Medio Ambiente y Recursos Naturales - Instituto Nacional de Geografía, Estadística e Informática- Programa de las Naciones Unidas para el Desarrollo, Distrito Federal.

Propín Frejomil, E., A. López López y A. Sánchez Crispín (2004), “Territorios preferenciales de los grupos hoteleros internacionales en América Latina y el Caribe a principios del Siglo XXI”, en: Investigaciones Geográficas, No. 53, pp. 122 - 140, Universidad Nacional Autónoma de México - Instituto de Geografía, Distrito Federal.

Rodríguez Aramberri, J. (2008), “Turismo y globalización: reflexiones críticas”, en: II Jornadas sobre Turismo y Sociedad, Instituto de Estudios Sociales Avanzados, Ministerio de Educación y Ciencia, Madrid.

Rodríguez Gómez, F. (1997), Medio ambiente, desarrollo y paisaje en las sociedades postindustriales, Universidad Complutense de Madrid, Madrid.

Rodríguez, V. y T. Warnes (2002), “Los residentes europeos mayores en España: repercusiones socioeconómicas y territoriales”, en: El campo de las ciencias y las artes, No. 139, pp. 123 - 148, Servicio de Estudios BBVA.

Sánchez, J. E. (2007), Pautas para la localización de las sedes de las grandes empresas y entornos metropolitanos, en: Revista EURE, Vol. XXXIII, No. 100, pp. 69 - 90, Pontificia Universidad Católica de Chile, Chile.

Santos, M. (2008), A Natureza do espaço, técnica e tempo, razão e emoção, Editora da Universidade do São Paulo, São Paulo.

Sauer, C. (1925), “The Morphology of Landscape”, en: Publications in Geography, Vol. III, No. 2, pp. 19 - 54, University of California Press, Berkeley.

Saxe-Fernández, J. (2006), "Libre mercado, seguridad y el nuevo anexionismo”, en: Observatorio Social de América Latina, Año VI, No. 18, pp. 297 - 303, CLACSO, Buenos Aires.

SECTUR (2002), Inversión turística privada 2002, Secretaría de Turismo, Distrito Federal.

SECTUR (2003), Inversión turística privada 2003, Secretaría de Turismo, Distrito Federal.

SECTUR (2004), Inversión turística privada 2004, Secretaría de Turismo, Distrito Federal.

SECTUR (2005), Inversión turística privada 2005, Secretaría de Turismo, Distrito Federal. 
SECTUR (2006), Inversión turística privada 2006, Secretaría de Turismo, Distrito Federal.

SECTUR (2007a), Inversión turística privada 2007, Secretaría de Turismo, Distrito Federal.

SECTUR (2007b), Programa Sectorial de Turismo 2007 - 2012, Secretaria de Turismo, Distrito Federal.

SECTUR (2008), La inversión privada identificada en el sector turístico: Padrón Nacional de Proyectos de Inversión, Secretaría de Turismo, Distrito Federal.

SEMARNAT (2005), Indicadores básicos de desempeño ambiental de México, Secretaría del Medio Ambiente y Recursos Naturales, Distrito Federal.

SER (2004), The SER International Primer on Ecological Restoration, Society for Ecological Restoration International Science \& Policy Working Group, Tucson.

Sessa, A. (1970), Tourism in developing countries, UNESCO, Paris.

Stonich, S. (1998), "Political Ecology of Tourism”, en: Annals of Tourism Research, Vol. 25, No. 1, pp. 25 - 54, Elsevier, Londres.

Tilley, C. (2006), “Introduction: Identity, Place, Landscape and Heritage”, en: Journal of Material Culture, Vol. 11, No. 1 \& 2, pp. 7 - 32, SAGE Publications, Londres.

Torres Bernier, E. (2003), "El turismo residenciado y sus efectos en los destinos turísticos”, en: Estudios Turísticos, No. 155 - 156, pp. 45 - 70, Ministerio de Economía y Turismo, Madrid.

Touraine, A. (2001), “El fin de la ola neoliberal”, en: Desigualdad y globalización: cinco conferencias, Universidad de Buenos Aires, Argentina.

UNESCO (1996), Angkor - Past, Present and Future, Cambodia Authority for the Protection and Safeguarding of the Angkor Region (APSARA), Paris.

Urry, J. (1990), “The Consumption of Tourism”, en: Sociology, Vol. 24, No. 1, pp. 23 - 35, SAGE Publications, Londres.

Valencia, M., y M. Ibarra (2006), El crecimiento de las ciudades. Su expansión hacia la periferia e interacción con el paisaje natural, Universidad Central de Chile, Santiago de Chile. 
Vera Rebollo, F. (1992), “Turismo y crisis agraria en el litoral alicantino”, en: Francisco Jurdao Arrones (ed.), Los mitos del turismo, Endymion, Madrid.

Wagley, C. y M. Harris (1974), "Una tipología de subculturas latinoamericanas”, en: C. Wagley (ed.), Estudios sobre el campesinado Latinoamericano: la perspectiva desde la antropología social, Ediciones Periferis, Buenos Aires. 